Peter Koller

\title{
Zur Kritik der libertären Eigentumskonzeption
}

Am Beispiel der Theorie von Robert Nozick

\begin{abstract}
Nozick's entitlement theory of justice is, besides Rawls's theory, one of the most widely discussed and intellectually most attractive conceptions within the field of contemporary political philosophy. Nozick's theory uses Locke's conception of the state of nature and of natural rights, and tries, starting from this point of view, to deliver a comprehensive systematisation of libertarian political ideals. This essay deals mainly with Nozick's conception of property rights. The argument is put forward that the concept of exclusive and unrestrictable ownership of which Nozick makes use, doesn't find any acceptable justification on the basis of his theory.
\end{abstract}

\section{Vorbemerkungen}

Der amerikanische Philosoph Robert Nozick hat in seinem 1974 erschienenen Buch „Anarchy, State and Utopia“" $(1974)^{1}$ eine Gerechtigkeitskonzeption entwickelt, die weit über die Grenzen der Berufsphilosophie hinaus Resonanz gefunden hat. Diese von Nozick als „Anspruchstheorie der Gerechtigkeit" ("entitlement theory") bezeichnete Konzeption, die das Ziel hat, eine freiheitliche Gerechtigkeitsvorstellung zu begründen, dürfte - zumindest für die nächste Zeit - gute Chancen haben, neben egalitären und utilitaristischen Gerechtigkeitskonzeptionen sowie auch neben der Theorie von John Rawls (1971) als eine der bemerkenswertesten theoretischen Leistungen im Umfeld der neueren politischen Philosophie angesehen $\mathrm{zu}$ werden. Diese Vermutung stützt sich vor allem auf den Umstand, daß es Nozick gelungen zu sein scheint, in seiner Theorie eine Reihe wohlbekannter, meist von konservativer Seite proklamierter Argumentationsmuster unter einem einheitlichen Gesichtspunkt zusammenzufassen und damit gleichsam ein „Paradigma" libertärer Philosophie wieder zu beleben, welches zu anderen philosophischen Ansätzen wirkungsvoll kontrastiert. Nozicks Theorie scheint die ideologische Position der freien Marktwirtschaft und des Unternehmertums in intellektuell anspruchsvoller Weise zu fundieren und kommt daher gewissen Interessen an der Propagierung eines Laisser-faire-Kapitalismus sehr gelegen.

In sachlicher Hinsicht verdient Nozicks Konzeption vor allem aus zwei Gründen Interesse: einmal, weil sie ein geläufiges Bestandselement unseres Alltagsverständnisses von Gerechtigkeit aufnimmt und konsequent zu einer allgemeinen Theorie sozialer Gerechtigkeit weiterzuführen versucht: die Vorstellung nämlich, daß Gerechtigkeit in der Achtung gewisser natürlicher und wohlerworbener Rechte bestehe. Zum zweiten ist Nozicks Theorie auch deshalb interessant, weil sie ein 
Paradigma der Sozialkontraktstheorie wiederbelebt, welches weitgehend in Widerspruch steht zu der von Rousseau und Kant vertretenen Vertragskonzeption, an die neuerdings John Rawls anknüpfte. Nozick greift im wesentlichen auf die Sozialvertragsvorstellung von John Locke zurück, vor allem auf dessen Konzept des Naturzustandes und der ursprünglichen Rechte des Menschen. Demgemäß hat der Sozialkontrakt bei Nozick - anders als bei Rawls - nicht unmittelbar die Funktion eines Ausgangspunktes für die Rechtfertigung von Gerechtigkeitsgrundsätzen, sondern nur die Aufgabe, die Grenzen staatlicher Eingriffe in die Rechte der Menschen zu bestimmen. Die Grundsätze der Gerechtigkeit ergeben sich bei Nozick vielmehr bereits aus dem Konzept der natürlichen Rechte des Menschen. Wie Locke vertritt er die Ansicht, daß diese ,,natürlichen“ oder ,ursprünglichen“ Rechte nicht erst aus dem Gesellschaftsvertrag resultieren, sondern den Menschen schon kraft ihrer Zugehörigkeit zur Menschengattung zustehen; sie bestehen bereits im Naturzustand.

Die Anspruchstheorie der Gerechtigkeit bildet nur einen Teil des Argumentationszusammenhanges, den Nozick in „Anarchie, Staat, Utopia“ entfaltet. Die Hauptthese dieses Buches besteht eigentlich darin, daß nur ein Minimalstaat oder - wie Nozick auch sagt - „Nachtwächterstaat“ moralisch gerechtfertigt ist; ein Staat also, der sich darauf beschränkt, das Leben, die Freiheit und das Eigentum der Bürger zu schützen, die Einhaltung von Verträgen zu garantieren, sowie Bedrohung von außen abzuwehren. Dagegen lasse sich jeder weitergehende Staat, so etwa vor allem ein Staat, der sich anmaßt, Umverteilungen vorzunehmen, nicht rechtfertigen. Nozick begründet diese Hauptthese in zwei Schritten:

1. In einem ersten Schritt versucht er nachzuweisen, daß sich aus der Anarchie (in Form von Lockes Naturzustand) zwangsläufig, d.h. ohne daß es irgendjemand beabsichtigt, ein Staat entwickelt. Im günstigen Fall kann sich diese Entwicklung - so meint er - in einer Weise vollziehen, die niemandes Rechte verletzt. Wenn diese Voraussetzung erfüllt ist, so erscheine das Gewaltmonopol eines solcherart zustandegekommenen Staates als gerechtfertigt; nur ein Minimalstaat könne diese Voraussetzungen erfüllen.

2. Darüber hinaus versucht Nozick - im zweiten Schritt - nachzuweisen, daß sich kein weitergehender Staat als der Minimalstaat rechtfertigen läßt. Seine Argumentation geht dabei dahin, daß die Gründe, die für einen weitergehenden Staat angeführt werden könnten, insbesondere auch die herkömmlichen Vorstellungen sozialer Gerechtigkeit, falsch sind. Zu diesem Zweck entwickelt er die Anspruchstheorie der Gerechtigkeit.

Obwohl für uns hier vor allem der zweite Teil von Nozicks Argumentationsstrategie von Interesse ist, ist es erforderlich, vorweg einige Bemerkungen zum Konzept der ,,natürlichen“ Rechte vorauszuschicken, von dem Nozick - mit Berufung auf Locke - von allem Anfang an ausgeht; im Anschluß daran werde ich in aller Kürze Nozicks Gerechtigkeitstheorie darlegen, um dann zu einer kritischen Analyse seiner Eigentumskonzeption überzugehen. 


\section{Nozicks Konzept der natürlicben Recbte}

Nozick geht mit Locke davon aus, daß die Menschen natürliche oder ursprüngliche Rechte haben, die sich aus der Vorstellung ergeben, die Menschen befänden sich anfänglich in einem Naturzustand, worin sie vollkommen frei sind, nach Gutdünken zu handeln und über ihre Person und ihre Besitztümer zu verfügen, ohne irgendeinen anderen Menschen um Erlaubnis bitten zu müssen. Die Menschen haben nach dieser Vorstellung ein natürliches Recht auf ihren Körper und auf ihre Arbeitsprodukte; ferner sind sie berechtigt, Verträge zu schließen. Sie sind dabei nur gebunden an das Naturrecht, welches besagt, daß niemand einen anderen an seinem Leben, seiner Gesundheit, seiner Freiheit oder seinem Eigentum schädigen darf und jeder die von ihm freiwillig eingegangenen Verträge einzuhalten hat. Gegen diejenigen, die Übergriffe gegen Rechte anderer begehen und anderen Schaden zufügen, dürfen die Menschen sich und andere verteidigen; die Geschädigten können von den Schädigern Wiedergutmachung fordern und darüber hinaus hat jedermann das Recht, Verstöße gegen jemandes Rechte zu bestrafen, damit sie künftig unterbleiben, aber nur insoweit, als die Strafe zum Zweck der Wiedergutmachung vergangener und der Prävention weiterer Übergriffe dient (vgl. 25).

Die private und persönliche Durchsetzung der Rechte im Naturzustand führt jedoch zu endlosen Fehden, da es keine klaren Möglichkeiten gibt, Streitigkeiten zu schlichten und zu beenden. Während Locke annimmt, daß sich die Menschen angesichts dieser Unzukömmlichkeiten des Naturzustandes in einem Sozialkontrakt einmütig einer mit Zwangsbefugnis ausgestatteten staatlichen Autorität unterwerfen, welche die Rechte der Menschen schützt und sozialen Frieden herstellt, stellt Nozick die These auf, daß sich eine staatliche Autorität ganz automatisch, auch ohne einen auf sie abzielenden Willensakt der Beteiligten, gleichsam durch einen Vorgang der unsichtbaren Hand, aus dem Naturzustand entwickelt.

Bei Locke leitet sich die Legitimität der staatlichen Gewalt aus der - wenn auch nur kontrafaktisch unterstellten - einmütigen Zustimmung aller Bürger her. Nozick, der auf diese Konstruktion verzichten möchte, geht dagegen davon aus, daß auch ein naturwüchsiger, ohne Zustimmung der Bürger entstandener Staat moralisch gerechtfertigt sein kann; Voraussetzung hierfür ist, daß sich die staatliche Gewalt aus dem Naturzustand auf eine Weise entwickelt, durch die niemand in seinen ursprünglichen und wohlerworbenen Rechten beeinträchtigt wird. Diese Voraussetzung ist nach Nozicks Auffassung dann erfüllt, wenn die Vorgänge, durch die aus dem Naturzustand ein Staat entsteht, gewisse moraliscbe Nebenbedingungen nicht verletzen. Die moralischen Nebenbedingungen schließen aus, daß die Unverletzlichkeit von Menschen irgendwelchen Zielen geopfert wird, wie moralisch wertvoll diese Ziele auch sein mögen. Sie sind Ausdruck des Kantischen Grundsatzes, daß die Menschen Zwecke sind und nicht bloß Mittel sein dürfen. Nozick:

„Die moralischen Nebenbedingungen für unser Handeln spiegeln, so behaupte ich, die Selbständigkeit unserer individuellen Existenz wieder, die Tatsache, daß es zwischen uns keinen 
moralischen Ausgleich geben kann: ein Leben wird nicht durch andere aufgewogen, so daß sich ein größeres gesellschaftliches Gesamtwohl ergäbe. Es ist nicht gerechtfertigt, einige um anderer willen zu opfern. Dieser Grundgedanke, daß es verschiedene Einzelmenschen mit einem je selbständigen Leben gibt, so daß keiner für andere geopfert werden darf, liegt den moralischen Nebenbedingungen zugrunde, führt aber auch, wie ich glaube, zu der freiheitlichen Nebenbedingung, die körperliche Ubergriffe gegen andere verbietet." (44)

Ein Staat kann nach Nozicks Dafürhalten daher nur insoweit moralische Legitimität für sich beanspruchen, als es gelingt zu zeigen, daß er aus dem Naturzustand hervorgehen kann, ohne moralische Nebenbedingungen zu verletzen, d.h. ohne in die Rechte von Menschen einzugreifen. Während Nozick nachweisen zu können glaubt, daß ein Minimalstaat, d.i. ein Staat mit Gewaltmonopol und geringfügigen Umverteilungswirkungen, die aus der Aufbringung der zum Schutz von Leben, Freiheit und Eigentum aller Bürger erforderlichen Steuerleistungen resultieren, auf moralisch zulässige Weise entstehen kann, ${ }^{2}$ meint er, daß jeder weitergehende Staat die Rechte der Menschen notwendig verletzen müsse, so vor allem ein Staat, dessen Aktivitäten auf eine wirkliche Umverteilung der Besitztümer hinausliefen. Als Grundlage dieser zweiten Behauptung dient ihm im wesentlichen die Anspruchstheorie der Gerechtigkeit.

\section{Die Anspruchstbeorie der sozialen Gerechtigkeit}

Die Gerechtigkeit der Verteilung von Besitztümern hängt nach Nozicks Auffassung von drei Umständen ab:

1. dem ursprünglichen Erwerb von Besitztümern (original acquisition of holdings), also der Aneignung herrenloser Gegenstände: hierzu bedarf es eines Grundsatzes der gerechten Aneignung;

2. der Übertragung von Besitztümern (transfer of holdings) von einer Person auf eine andere: hierzu bedarf es eines Grundsatzes der gerechten Übertragung; sowie

3. der Möglichkeit der Korrektur früherer Verletzungen der ersten beiden Grundsätze der Gerechtigkeit (hierfür braucht man einen Grundsatz der Berichtigung ungerechter Besitzverhältnisse).

In einer Welt, in der es keine Ungerechtigkeiten gäbe, wäre die Verteilung des Besitzes - so sagt Nozick - allein nach den folgenden drei Grundsätzen geregelt:

„1.Wer ein Besitztum im Einklang mit dem Grundsatz der gerechten Aneignung erwirbt, hat Anspruch auf dieses Besitztum.

2. Wer ein Besitztum im Einklang mit dem Grundsatz der gerechten Übertragung von jemandem erwirbt, der Anspruch auf das Besitztum hat, der hat Anspruch auf das Besitztum.

3. Ansprüche und Besitztümer entstehen lediglich durch (wiederholte) Anwendung der Regeln 1 und 2.

Der vollständige Grundsatz der Verteilungsgerechtigkeit würde einfach besagen, eine Verteilung sei gerecht, wenn jeder auf die Besitztümer Anspruch hat, die ihm bei der Verteilung zugehören." (144) 
Da diese Grundsätze jedoch nicht immer strikt eingehalten werden, muß es für jene Fälle, in denen eine Situation nicht im Einklang mit ihnen entstanden ist (wenn z.B. ein Besitztum durch Diebstahl, Betrug u.dgl. angeeignet wurde), einen Grundsatz geben, der diese Ungerechtigkeiten zu korrigieren erlaubt: einen Berichtigungsgrundsatz. Nach diesem Grundsatz ist - so schlägt Nozick vor jene Verteilung herzustellen, die eingetreten wäre, wenn die Ungerechtigkeit nicht geschehen wäre.

Damit sind die Grundbestandteile der von Nozick vertretenen, von ihm „,Anspruchstheorie" genannten Gerechtigkeitstheorie angegeben. Er faßt die Grundzüge dieser Theorie wie folgt zusammen:

„Der Besitz eines Menschen ist gerecht, wenn dieser auf ihn im Sinne der Grundsätze der gerechten Aneignung und Übertragung oder der Berichtigung von Ungerechtigkeiten (im Sinne der ersten beiden Grundsätze) einen Anspruch hat. Ist der Besitz jedes einzelnen gerecht, so ist die Gesamtmenge (die Verteilung) der Besitztümer gerecht.“(146)

Nozick unterläßt es allerdings, diese allgemeinen Richtlinien auf eine Weise zu konkretisieren, welche die Grundsätze der Erstaneignung, der Übertragung und der Korrektur von Ungerechtigkeiten inhaltlich genau bestimmen würde. Er geht aber davon aus, daß es möglich ist, diese Grundsätze im Detail auszuführen, und daß es genau diese Grundsätze wären, nach denen sich die Beurteilung der Verteilungsgerechtigkeit allein zu richten hat (vgl. 146).

Ein neuralgischer Punkt der Anspruchstheorie ist - wie Nozick selbst zugesteht - die Frage der gerechten Aneignung, von der ja letztlich auch die Gerechtigkeit aller auf die Aneignung folgenden Vorgänge abhängt. Da Nozick annimmt, die ursprüngliche Aneignung eines Gegenstandes begründe ein volles, also ausschließliches und unwiderrufliches Eigentumsrecht an diesem Gegenstand, kommt dieser Frage bei ihm besondere Relevanz zu. Nun, welches Konzept der ursprünglichen Aneignung bietet er an, aus dem ein so weitgehendes Besitzrecht wie das volle Eigentum abgeleitet werden könnte?

Nozick knüpft an die Aneignungstheorie von Locke an, der meinte, daß ein Eigentumsrecht an einem herrenlosen Gut dadurch entsteht, daß man es bearbeitet. Locke ging dabei offenbar von der Vorstellung aus, daß etwas durch die Bearbeitung besser und wertvoller wird. Wie Nozick bemerkt, stellt sich dann allerdings immer noch die Frage, warum man auf den ganzen Gegenstand und nicht bloß auf den Mehrwert, der durch die Arbeit entstanden ist, einen Anspruch haben sollte. Denn es erscheine wohl kaum plausibel, aus der Verbesserung eines Gegenstandes das volle Eigentum abzuleiten, wenn der Vorrat an verbesserungsfähigen herrenlosen Gegenständen begrenzt ist. Daher stellte Locke die Bedingung auf, daß die Verbesserung eines Gegenstandes nur dann ein Eigentum daran begründe, wenn für andere ,genug und gleich Gutes“ übrig bleibe. Mit dieser Bedingung wollte Locke offensichtlich verhindern, daß durch die Aneignung herrenloser Gegenstände die Lage anderer verschlechtert wird, denen es nach der Aneignung ja nicht mehr freisteht, diese zu gebrauchen. 
Man könnte nun allerdings meinen, daß jede Aneignung die Lage der anderen irgendwie verschlechtert, zumindest insoweit, als diese infolge der Aneignung-in ihrer Möglichkeit beschränkt sind, sich etwas anzueignen, was sich jemand bereits zuvor angeeignet hat. Wenn aber jede Aneignung die Lage anderer irgendwie verschlechtert, dann wäre nach dieser Interpretation der Locke'schen Bedingung eine Aneignung überhaupt niemals zulässig. Dazu Nozick:

„Doch diese Argumentation ist vorschnell. Jemand kann durch eine Aneignung seitens eines anderen auf zweierlei Weise schlechter gestellt werden: erstens dadurch, daß er die Möglichkeit verliert, seine Lage durch eine bestimmte oder eine beliebige Aneignung zu verbessern; zweitens dadurch, daß er etwas nicht mehr (ohne Aneignung) freizügig nutzen kann. Eine strenge Bedingung, daß kein anderer durch eine Aneignung schlechter gestellt werden darf, würde die erste Weise ausschließen, wenn die Einschränkung der Möglichkeiten auf keine andere Art ausgeglichen wird, und auch die zweite. Eine schwächere Bedingung würde nur die zweite, nicht die erste Weise ausschließen.“(165)

Nozick hält die schwächere Bedingung für vollkommen ausreichend, um die Interessen derjenigen $\mathrm{zu}$ wahren, die sich aufgrund einer vorhergehenden Aneignung durch einen anderen etwas nicht mehr aneignen können, denn es könne sich schließlich niemand darüber beklagen, daß er sich etwas nicht mehr aneignen kann, wenn er es doch immerhin noch frei nutzen könne. Er meint daher, daß es genügt, die ursprüngliche Aneignung von Gütern durch folgende schwache Version der Locke' schen Bedingung zu beschränken: Ein Vorgang, der zu einem dauernden, erblichen Eigentumsrecht an einer bisher herrenlosen Sache führt, ist zulässig, wenn er die Lage anderer nicht dadurch verschlechtert, daß sie die Sache nicht mehr frei nutzen können. Jedenfalls müsse jede brauchbare Theorie der Aneignung eine Bedingung genau dieser Art enthalten.

Obwohl Nozick es unterläßt, seine Konzeption der Aneignung im Detail auszuführen und im einzelnen zu begründen, zögert er nicht zu erklären, ein freies Marktsystem mit Privateigentum genüge ohne Zweifel der von ihm vorgeschlagenen schwächeren Version der Locke'schen Bedingung. Immerhin räumt er ein, daß diese die Aneignung limitierende Bedingung teilweise auch die Möglichkeiten späterer Übertragungen einschränkt, u.zw. insofern, als es zu keinen Eigentumsballungen kommen darf, die es zulassen, daß die Eigentümer alle anderen vom Gebrauch bestimmter Gegenstände ausschließen können. Allerdings gilt die Bedingung, daß die Aneignung andere hinsichtlich ihrer Möglichkeit der Nutzung nicht schlechter stellen darf, nach Nozicks Auffassung nur für Sachen, die zur Aneignung bereitstehen und nicht für lebensnotwendige Sachen schlechthin: ein medizinischer Forscher z. B., der eine neue Heilsubstanz hergestellt hat und der diese nur unter seinen Bedingungen verkaufen will, verschlechtert Nozick zufolge nicht die Lage anderer, indem er ihnen etwas entzöge, was sie sonst nutzen könnten; die anderen könnten sich ja selbst die Materialien aneignen, die der Forscher benutzt hat. Jedenfalls biete die Sicherung der allgemeinen Nutzungsmöglichkeit von angeeigneten Sachen - so glaubt er - keinen wie immer gearteten Anhaltspunkt für eine über den Minimalstaat hinausgehende Staatstätigkeit (vgl. $167 \mathrm{ff})$. 


\section{Die Rechte des Menschen als negative Rechte}

Nozick geht davon aus, daß die Menschen von Geburt an mit gewissen natürlichen moralischen Rechten ausgestattet sind, die aus der Vorstellung des Naturzustandes gewonnen werden. Sehen wir uns etwas genauer an, wie er diese natürlichen Rechte bzw. Ansprüche, die gleichsam den Angelpunkt der Rechtfertigung seiner Gerechtigkeitsvorstellung bilden, im einzelnen bestimmt.

Nozick übernimmt im wesentlichen die Locke'sche Vorstellung, daß die Menschen als freie und gleiche Wesen geboren werden, die - körperliche und geistige Reife vorausgesetzt - durch keinerlei natürliche Abhängigkeiten gebunden sind und die alle grundsätzlich das gleiche Recht haben, ihr Leben und ihre Freiheit zu verteidigen und sich zur Fristung ihres Lebens die Umwelt zueigen zu machen. Jeder Mensch hat demnach ein subjektives moralisches Recht auf Leben, Freiheit und Eigentum. Diese Vorstellung scheint zunächst intuitiv durchaus einleuchtend und wir wollen sie für die Zwecke der weiteren Diskussion akzeptieren.

Mit der Annahme von subjektiven moralischen Rechten der Einzelmenschen allein ist allerdings keineswegs auch schon ein ,objektiver", d.h. allgemein anwendbarer Moralkodex gegeben, der das Handeln der Menschen in widerspruchsfreier und hinreichend bestimmter Weise regeln würde. Denn einerseits befinden sich subjektive moralische Rechte verschiedener Individuen zueinander häufig im Widerspruch, und andererseits sind die subjektiven moralischen Rechte zu unterbestimmt, um schon eine generelle Norm des moralisch angemessenen gegenseitigen Verhaltens zu ergeben. Daher gilt es, einen objektiven, für alle verbindlichen Kodex moralischer Normen zu etablieren, der die widerstreitenden Ansprüche der Einzelnen in ihrem Verhältnis zueinander verträglich macht und deren Unbestimmtheit beseitigt. Da die Harmonisierung gegensätzlicher Ansprüche nur durch einen solchen Moralkodex erfolgen kann, ergeben sich erst aus diesem diejenigen subjektiven moralischen Rechte und Pflichten, die das gegenseitige Verhalten der Individuen in anwendbarer und handhabbarer Weise bestimmen können. ${ }^{3}$ Das Geschäft der moralischen Rechtfertigung besteht demnach in der Hauptsache darin, allgemeine Normen zu finden, die subjektive Zwecke und Interessen in annehmbarer Weise verträglich machen. Mit der Statuierung subjektiver Ansprüche allein ist noch nichts getan, wenn man nicht zugleich auch zeigt, daß die ihnen zugrundeliegenden Zwecke und Interessen untereinander vereinbar, also logisch verträglich, wie auch von einem allgemeinen und unparteiischen Standpunkt aus betrachtet ausgeglichen sind (ähnlich Nagel 1975, 140).

Die Lösung, die Nozick für dieses Problem anbietet, stellt zwar die formale Konsistenz der subjektiven Rechte der verschiedenen Einzelpersonen her, führt aber nichtsdestoweniger zu einem vom moralischen Standpunkt gänzlich unannehmbaren Beziehungsverhältnis der moralischen Rechte der Menschen untereinander. Seine Lösung besteht nämlich darin, die natürlichen moralischen Rechte der Menschen ganz eng zu fassen, sie als rein negative Rechte zu konzipieren: diese Rechte schützen jedermann nur gegen gewaltsame Übergriffe auf Leben, Freiheit und Besitztum, verleihen jedoch niemandem auch nur den geringsten posi- 
tiven Anspruch gegen andere, wenn es darum geht, diese Güter überhaupt erst für alle Menschen sicherzustellen.

Diese enge Konzeption der natürlichen Rechte erscheint alles andere als einleuchtend, wenn man in Erwägung zieht, was sie eigentlich bedeutet. Peter Singer hat diesen Punkt sehr klar herausgearbeitet (Singer 1978, 209):

\begin{abstract}
„Man betrachte zum Beispiel das Recht auf Leben. So wie man dieses Recht im allgemeinen versteht, beinhaltet es nicht nur das Recht, von anderen nicht getötet zu werden, sondern auch das Recht auf Nahrung, wenn wir verhungern, während andere im Überfluß leben, sowie das Recht auf ein Minimum an medizinischer Versorgung, wenn die Gesellschaft, in der wir leben, es sich leisten kann, sie sicherzustellen. Wenn eine Gesellschaft es zuläßt, daß die Menschen den Hungertod sterben, wenn es ringsum mehr als genug Nahrung gibt, um alle zu ernähren, oder wenn sie es zuläßt, daß die Menschen an Krankheiten sterben, weil sie zu arm oder zu unwissend sind, um eine einfache und billige Injektion zu bekommen, dann würden wir sicherlich nicht meinen, daß in dieser Gesellschaft das Recht auf Leben in hohem Maße respektiert wird. Mit anderen Worten, das Recht auf Leben wird im allgemeinen ebenso als Recht auf Unterstützung (right of recipience) wie als Recht gegen Ubergriffe verstanden."
\end{abstract}

Da das Konzept der natürlichen moralischen Rechte des Menschen bei Nozick den Rahmen für den Erwerb von Rechten und Ansprüchen überhaupt abgibt, überträgt sich der restriktive moralische Gehalt, mit dem er dieses Konzept ausstattet, in epidemischer Weise auch auf alle, erworbenen Rechte und Ansprüche, die er den Menschen als moralisch gerechtfertigte zugesteht. Die verheerenden Implikationen dieses Konzepts der natürlichen Rechte treten besonders deutlich hervor in Nozicks Theorie der Eigentumsrechte, die im Rahmen seiner Konzeption eine zentrale Stellung einnimmt und der ich mich nun zuwenden möchte.

\title{
5. Nozicks Konzeption der Eigentumsrechte: eine Apologie des Besitzindividua-
} lismus

Die Vorstellung eines ausschließlichen und unwiderruflichen Eigentumsrechts an Besitztümern spielt in Nozicks Konzeption der Gerechtigkeit eine tragende Rolle. Trotzdem unterläßt er es, auch nur den Ansatz einer systematischen Rechtfertigung dieser Vorstellung zu liefern. Stattdessen begnügt er sich damit, auf die Theorie des Eigentumserwerbs von John Locke hinzuweisen. Wir können daher davon ausgehen, daß Nozick - abgesehen von einigen ausdrücklichen Einschränkungen - Lockes Konzeption im wesentlichen übernimmt.

Locke geht davon aus, Gott habe die Welt allen Menschen gemeinsam gegeben, damit sie sie zur Fristung ihres Lebens und zur Vermehrung ihrer Wohlfahrt nutzen. ${ }^{4}$ Niemand habe über die Umwelt und die Früchte, die sie auf natürliche Weise hervorbringt, ursprünglich ein alleiniges Verfügungsrecht, das die anderen ausschlösse. Da jedoch die Welt den Menschen zu ihrem Gebrauch und Vorteil gegeben sei, sei es notwendig, irgendeine Form des Eigentumserwerbs an den Gegenständen der Umwelt vorzusehen; denn erst dann, wenn einem etwas als Ei- 
gentum gehöre, so daß kein anderer ein Recht darauf habe, sei es ihm zur Erhaltung seines Lebens von Nutzen. Zunächst habe jeder Mensch ein Eigentum, ein ausschließliches Verfügungsrecht an seiner eigenen Person und an seiner Arbeitskraft. Ferner: da die Arbeit das Eigentum des Arbeitenden sei und niemand außer ihm selbst ein Recht auf irgendetwas habe, was einmal mit seiner Arbeit verbunden ist, erwerbe der, der seine Arbeit mit Gegenständen der natürlichen Umwelt vermische, ein Eigentum an diesen Gegenständen - sofern Gegenstände gleicher Qualität für die anderen noch in ausreichender Menge vorhanden sind.

Nozick scheint mit dieser Rechtfertigung nicht ganz einverstanden zu sein; er bemerkt nämlich mit Recht, daß sie es offenläßt, durch welche Art der Arbeit Eigentum erworben werde und welche Gegenstände das Eigentumsrecht umfasse. Allerdings gibt auch er keine Antworten auf diese Fragen. Jedenfalls stimmt er mit Locke darin überein, daß man an allen Gegenständen Eigentum erwerben kann, von deren Nutzung man andere ausschließen kann: dazu gehören nicht nur die Früchte und die beweglichen Mittel der Arbeit (wie z. B. Arbeitswerkzeuge), sondern auch die natürlichen Ressourcen der Arbeit, so vor allem Grund und Boden und Bodenvorräte.

Wenn wir vorläufig einmal darüber hinwegsehen, daß sich die Annahme eines so weitgehenden Eigentumsrechts aus den Locke'schen Prämissen keineswegs zwingend ergibt, müssen wir Locke zugutehalten, daß er sich gerade durch den Gedanken, die Welt sei allen Menschen zur Sicherung ihrer Subsistenz gegeben, veranlaßt sah, die Aneignungsmöglichkeiten durch folgende drei Bedingungen zu beschränken:

1. Jeder darf sich nur soviel aneignen, daß für die anderen genug von derselben Qualität übrigbleibt;

2. jeder darf sich nur soviel aneignen, wie er verzehren kann; und

3. jeder darf sich nur soviel Boden aneignen, wie er bearbeiten kann. (Siehe Locke 1690,2 . Abhandlung, $\$ \$ 31-34,218$ ff.)

Obwohl Locke diesen Bedingungen selbst nicht wirklich konsequent Rechnung getragen hat und sie - vermutlich in der Absicht, die Besitzverhältnisse im England des 17. Jahrhunderts zu rechtfertigen - im Verlauf seiner weiteren Erörterungen mitunter durch windige Ad-hoc-Konstruktionen eingeschränkt hat, zeigen sie doch, daß er bemüht war, das Konzept der Eigentumsrechte mit dem Bedarf der Menschen einerseits und mit der gleichen Freiheit des Eigentumserwerbs für alle andererseits in Übereinstimmung zu bringen.

Demgegenüber erscheint das Eigentumsrecht bei Nozick als etwas, das mit dem Bedarf der Menschen so gut wie nichts zu tun hat. Es geht ihm ausschließlich um die Freiheit, jedoch nicht um die Freiheit aller Menschen, sondern nur derjenigen, die bereits über Eigentum verfügen. Nozick übernimmt zwar Lockes 1. Bedingung, schränkt sie aber dahingehend ein, daß man sich Gegenstände aneignen kann, solange den anderen noch Gegenstände dieser Art verbleiben, die sie - wenn auch ohne Aneignung - frei nutzen können; und er meint, daß der freie Markt die freie Nutzung von Gegenständen jeglicher Art sowieso verbürgt. Es fällt schwer, die- 
sen Gedanken nachzuvollziehen, weshalb es vielleicht zweckmäßig ist, sich Nozicks Konzeption der Aneignung an einem Beispiel zu veranschaulichen.

Nehmen wir an, es gäbe in der Nähe unseres Wohnortes einen See, der noch niemandem gehört und den wir hin und wieder zum Baden benutzen. Nozick scheint zu meinen, es bestehe kein Grund, diesen See als öffentliches Gut zu betrachten, sondern wir könnten ebensogut hingehen und uns die Ufergrundstücke aneignen. Wir müßten dabei nur dafür Sorge tragen, daß diejenigen, die den See gelegentlich zum Baden benutzt haben, das auch weiterhin tun können. Wir könnten das etwa dadurch bewerkstelligen, daß einer von uns auf seinem Grundstück eine öffentliche Badeanstalt errichtet, die den anderen gegen einen erschwinglichen Eintrittspreis offensteht.

Gegen dieses Beispiel könnte möglicherweise gesagt werden, es sei mit Absicht so gewählt, um Nozicks Version von Lockes erster Bedingung als unvernünftiger erscheinen zu lassen, als sie es wirklich ist. Natürlich gibt es auch Beispiele, in denen Nozicks Vorstellung, daß der freie Markt ohnehin die freie Nutzung allgemein begehrter Gegenstände gewährleistet und daß daher dem Eigentumserwerb in einem freien Marktsystem - zumindest unter normalen Bedingungen keinerlei Grenzen gesetzt zu sein brauchen, weniger absurd erscheint. Da Nozick jedoch für seine Konzeption der Aneignung immerhin allgemeine Geltung beansprucht, muß er wohl der Ansicht sein, Gegenstände wie Berge, Seen, Rohstoffe u.dgl. ließen sich in ähnlicher Weise aneignenen wie die wildwachsenden Früchte der natürlichen Umwelt und die Hervorbringungen der eigenen Arbeit. Überdies ist er der Meinung, die Aneignung verschaffe den Nutznießern ein unwiderrufliches Eigentumsrecht an den angeeigneten Gegenständen und niemand habe ein Recht, sie ihnen wegzunehmen oder sie in ihrer Verfügungsgewalt zu beschränken.

Nun fragt sich natürlich, wodurch denn ein so weitgehendes Eigentumsrecht, welches einzuschränken keinerlei Umstände zu rechtfertigen vermögen, begründet werden könnte. Nozick beantwortet diese Frage nicht. Veranschaulichen wir uns dieses Problem wieder an einem Beispiel:

Eine Gruppe von Familien siedelt sich in einem unbewohnten Gebiet an. Ihre Wirtschaftsweise sei die einer bäuerlichen Subsistenzwirtschaft. Um jeder Familie die Möglichkeit der Bedarfsdeckung zu geben, teilen sie den Boden auf und kommen überein, daß jeder Familie ein ausschließliches Eigentumsrecht an dem ihr zugeteilten Boden zusteht. Nach und nach treten sie in Tauschbeziehungen ein, es kommt zu einer Art der Arbeitsteilung zwischen ihnen und es ergeben sich gewisse technische Neuerungen. Sie entdecken Arbeitstechniken, die die Arbeitsproduktivität wesentlich steigern. Die Entwicklung einiger dieser Arbeitstechniken hängt ab von einem Rohstoff, den man aus dem Boden gewinnen kann. Nun stellt sich heraus, daß sich im Gebiet einer der Familien Bodenvorräte mit diesem Rohstoff befinden, während die anderen Familien keine derartigen Bodenvorräte haben. Die Frage, die sich angesichts dieser Sachlage stellt, ist natürlich die, ob die Familie, auf deren Boden sich der Rohstoffvorrat befindet, ein ausschließliches Eigentumsrecht auf diesen Vorrat beanspruchen kann oder nicht. Ist die 
ursprüngliche Aufteilung des Bodens, die im Hinblick auf eine ganz anders geartete wirtschaftliche Bedarfsbefriedigung vorgenommen wurde, unantastbar oder hat sie sich gar nicht auf die unter der Erdoberfläche verborgenen Bodenvorräte bezogen? Was könnte dafür sprechen, daß die Familie durch die usprüngliche Verteilung ein unwiderrufliches Eigentumsrecht auf den Boden, einschließlich der Bodenschätze, die er birgt, erworben hat? Offensichtlich dies, daß sie ihren Lebensplan auf die Bearbeitung dieses Bodens hin orientiert hat und daß Verfügungsrechte - wenn sie ihren Sinn erfüllen sollen - eine gewisse Sicherheit verschaffen müssen, daß einem nicht das, was man rechtmäßig erworben hat, willkürlich weggenommen werden kann. Es ist jedoch nicht zu sehen, daß daraus die Notwendigkeit eines ausschließlichen und unwiderruflichen Eigentumsrechts an irgendeinem Gegenstand folgen sollte.

Nozicks Irrtum liegt - worauf vor allem Cheyney C. Ryan hingewiesen hat hauptsächlich darin, daß er den Begriff des Besitztums, also dessen, was jemand hat (holdings), mit dem Begriff des Eigentums (ownership) konfundiert (siehe Ryan 1976/77, $130 \mathrm{ff}$; ähnlich auch Arrow 1978,276f). Daraus, daß es zweckmäßig ist, Rechte zu etablieren, die Menschen berechtigen, über bestimmte Gegenstände zu verfügen oder die Befugnisse gewisser Positionen auszuüben, leitet sich keineswegs schon die Berechtigung der Rechtsinhaber ab, mit diesen Gegenständen zu machen, was sie wollen, etwa ihre Amtsbefugnisse frei zu tauschen oder sie auf ihre Kinder zu vererben. Ryan erwähnt als Beispiel das Universitätssystem mit seinen Dienstposten für Universitätslehrer: obwohl es zweifelsohne erforderlich ist, den Inhabern dieser Positionen größtmögliche Freizügigkeit einzuräumen, Richtung und Inhalte ihrer Lehr- und Forschungstätigkeit selbst zu bestimmen, so wäre es doch widersinnig, ihnen auch das Recht zu geben, ihre Positionen zu verkaufen oder sie an ihre Kinder zu vererben. Wenn man nicht von vornherein unterstellt, daß alle unsere Rechte den Charakter von Eigentumsrechten haben, dann ist es auch nicht richtig zu sagen, die Beschränkung von Rechten auf bestimmte Befugnisse bedeute notwendig eine Verletzung persönlicher Freiheit; weil ja nicht ohne weiteres angenommen werden kann, daß uns diese Freiheit überhaupt zusteht (so auch Thomson 1977, $47 \mathrm{ff}$ ).

Das bedeutet natürlich nicht, daß es keine guten Gründe dafür gibt, unter bestimmten Bedingungen ein weitgehendes Eigentumsrecht an gewissen Gegenständen zu etablieren, etwa an solchen Gegenständen, die die Menschen zu Erhaltung ihrer Existenz unbedingt brauchen. Wenn man, wie noch Locke, das Eigentumsrecht als eine Institution versteht, die der Erhaltung der Existenz und der Steigerung der Wohlfahrt gleicher und freier Individuen dienen soll, dann besteht allerdings kein Grund, eine einmal vorgenommene Aufteilung des Besitztums als unwiderruflich zu betrachten. Katastrophen, Änderungen der Wirtschaftsweise oder auch eine unvorhergesehene Dynamik der aus einer Aufteilung des Besitzes resultierenden Austauschbeziehungen können eine zeitweilige Suspendierung oder überhaupt eine Aufhebung alter Eigentumsrechte begründen. Sicherlich aber ist es so, daß ein derartiger Eingriff in wohlerworbene Verfügungsrechte nicht willkürlich vorgenommen werden darf, sondern im Hinblick auf den 
Bedarf der Menschen und auf die Wahrung gleicher Lebenschancen gerechtfertigt werden können muß.

Ist schon Nozicks Konzeption der Aneignung (des ursprünglichen Erwerbs) eigentumsartiger Besitzrechte verfehlt, seine Theorie der Übertragung (des abgeleiteten Erwerbs) von Rechten ist es nicht minder. Wie wir gesehen haben, ist das Argument, auf dem Nozicks Konzeption der Gerechtigkeit als der Ausübung natürlicher und/oder wohlerworbener Rechte beruht, dieses:

„Alles, was aus gerechten Verhältnissen auf gerechte Weise entsteht, ist selbst gerecht.“ (144)

Nach Nozicks Auffassung entsteht ein Zustand aus einem anderen auf gerechte Weise genau dann, wenn dadurch moralische Nebenbedingungen nicht verletzt werden. Und da diese - wie er meint - immer dann gewahrt sind, wenn der Übergang aufgrund freiwilliger Transaktionen zwischen denjenigen Personen erfolgt, deren Rechte durch den Übergang berührt sind, muß er auch den folgenden Satz für wahr halten (vgl. Cohen 1977, 7):

„Alles, was aus gerechten Verhältnissen als Ergebnis freiwilliger Transaktionen zwischen allen in ihren Rechten betroffenen Personen entsteht, ist selbst gerecht."

Wenn dieser Satz richtig wäre, dann hätten wir Rechte und Ansprüche, die von ihren berechtigten Inhabern im Wege freiwilliger Übertragungsakte erworben wurden, immer auch als moralisch gerechtfertigt anzusehen. Das tun wir jedoch häufig nicht, und, so meine ich, in vielen Fällen mit gutem Grund. Nozicks Auffassung, freiwillige Transaktionen reichten hin, um die moralische Zulässigkeit von Rechten bzw. daraus resultierenden Pflichten sicherzustellen, hält denn auch einer kritischen Prüfung nicht stand. Unter anderem sprechen folgende Argumente gegen sie:

1. Zur Begründung seiner Doktrin beruft sich Nozick auf die zweite Formulierung von Kants Kategorischem Imperativ, die von uns verlangt, unsere Mitmenschen niemals nur als Mittel, sondern immer zugleich auch als Zweck zu behandeln. ${ }^{5}$ Angesichts der Implikationen von Nozicks Konzept moralischer Rechte klingt das wie ein schlechter Witz. Denn nach seinem Verständnis würde jede Art der Behandlung anderer diesem Prinzip Genüge tun, sofern sie nur mit deren freiwilligem Einverständnis geschieht, gleichgültig unter welchen Bedingungen die Betroffenen ihr Einverständnis erklären. So gesehen, verletzt Shylocks Forderung gegen Antonio, diesem bei lebendigem Leibe ein Pfund Fleisch nächst dem Herzen herausschneiden zu dürfen, ebensowenig das Gebot, andere immer auch als Zweck zu achten, wie die Versklavung von Menschen, die sich selbst der Sklaverei unterwerfen, oder der Zustand, daß kleine Kinder zum Schaden ihrer Gesundheit schwere Fabriksarbeit verrichten müssen, um die Hungerlöhne ihrer Eltern aufzubessern. Die Meinung, daß all das der Würde des Menschen entspreche, muß in hohem Maße als absurd anmuten, wenn der Begriff der menschlichen Würde 
irgendeinen vernünftigen Inhalt haben soll. Obschon Kants Prinzip natürlich die Forderung miteinschließt, daß wir in die Rechte anderer Personen nicht einfach ohne ihre Zustimmung eingreifen dürfen (weil wir sie sonst als bloße Mittel gebrauchten), erlegt es uns ebenso die Pflicht auf, andere so zu behandeln, daß sie gleich uns ihren eigenen Willen überhaupt geltend machen können, um sich nicht als bloßes Mittel benutzen lassen zu müssen. ${ }^{6}$ Bereits dieser Hinweis reicht aus, um Nozicks Annahme zu entkräften, daß ein System, in dem die Menschen ihre wohlerworbenen Ansprüche und Besitztümer nur im Wege freiwilliger Transaktionen übertragen, zwangsläufig dem Kantischen Grundsatz entspreche und damit notwendig die moralischen Nebenbedingungen erfülle. Die moralische Zulässigkeit sozialer Zustände läßt sich eben nicht allein daran festmachen, daß sie durch freiwillige Transaktionen zustandegekommen sind, sondern es kommt auch darauf an, ob die Randbedingungen, unter denen diese Transaktionen stattgefunden haben, ihrerseits moralisch zulässig waren. Wenn sich etwa in den gesellschaftlichen Verhältnissen krasse Ungleichheiten von Macht und Besitz manifestieren, die einigen Personen gar keine Chance einer entsprechenden Vertretung ihrer elementaren Zwecke einräumen und ihnen daher das Einverständnis zu unvorteilhaften Transaktionen gleichsam abnötigen, dann verletzt ein derartiger Zustand moralische Nebenbedingungen um nichts weniger als bestimmte Formen manifesten Zwangs.

2. Die Verhandlungsmacht, über die die Individuen in Transaktionen verfügen, und damit auch ihre Chance, den Inhalt freiwilliger Vereinbarungen nach ihren Interessen $\mathrm{zu}$ bestimmen, ist ferner in wesentlichem Maße durch die institutionellen Regeln bedingt, unter denen sich die Übertragungsakte vollziehen. Daher unterliegen auch diese Regeln der moralischen Kritik (so auch Scanlon 1976/77,14). Nehmen wir zum Beispiel den Arbeitsmarkt: Wenn es institutionelle Beschränkungen der Kinderarbeit, Schutz der Frauenarbeit und Vorsorge für kranke oder alte Arbeiter gibt, dann können die Arbeiter Arbeitsverträge mit für sie erheblich vorteilhafteren Bedingungen durchsetzen, als wenn diese Beschränkung nicht besteht. Man könnte vielleicht meinen, diese Beschränkungen brächten eine künstliche Verknappung des Angebots an Arbeitskraft und bedeuteten einen willkürlichen Eingriff in das freie Marktgeschehen. Wie jedoch die Geschichte der sozialen Bewegung zeigt, hat das Fehlen solcher institutioneller Schutzmaßnahmen eine Schwächung der Verhandlungsposition der Arbeiter zur Folge, aus der heraus sie genötigt sind, nolens volens in Arbeitsbedingungen einzuwilligen, die - wenn die Forderung der Achtung der menschlichen Würde nicht vollends zur Leerformel verkommen soll - jeglicher Menschenwürde Hohn sprechen. Nozick kann daher nicht recht haben, wenn er meint, der Moral sei allein schon durch die Freiwilligkeit der Transaktionen zwischen den Individuen Genüge getan, ohne Rücksicht darauf, ob die institutionellen Rahmenbedingungen des Marktgeschehens auch einen fairen Interessenausgleich zwischen den Verhandlungspartnern gewährleisten. 
3. Aber selbst die Annahme, daß wir nur Übertragungsvorgänge haben, die ausgehend von einer anfänglich gerechten Situation - unter gerechten institutionellen Rahmenbedingungen stattfinden und die Zwecke aller Beteiligten gebührend berücksichtigen, läßt noch nicht den Schluß auf die Gerechtigkeit der sich aus diesen Übertragungsakten ergebenden Verhältnisse zu. Dieser Schluß enthält nämlich - worauf G. A. Cohen aufmerksam gemacht hat - die stillschweigende Unterstellung, daß die Menschen bei Übertragungsvorgängen vollkommen oder zumindest weitgehend rational handeln und überdies die Folgen ihres Handelns vollständig überblicken können (siehe zum folgenden: Cohen 1977, 7 ff). Nur wenn jeder Beteiligte seine wohlerwogenen Absichten im Bewußtsein der vollen Konsequenzen seines Tuns mit den bestmöglichen der ihm verfügbaren Mittel zu verfolgen imstande ist, kann man wirklich sagen, niemand habe einen guten Grund, sich später über die Ergebnisse der von ihm mitbeschlossenen Transaktionen zu beklagen. Nozick selbst gesteht das mehr oder minder zu, wenn er meint:

„Immerhin ist zuzugeben, daß man nicht so recht zufrieden wäre, wenn die Menschen Eigentumsübertragungen stets nur aus irrationalen oder willkürlichen Gründen vornähmen. (Man stelle sich vor, die Menschen entschieden stets mittels eines Zufallsmechanismus, welche Besitztümer sie wem übertragen wollen.) Man ist eher geneigt, an die Gerechtigkeit eines reinen Anspruchsystems zu glauben, wenn die meisten Übertragungen aus Gründen heraus erfolgen. Das bedeutet nicht unbedingt, daß alle die Besitztümer verdienten, die sie erhalten. Es bedeutet nur, $\mathrm{da} ß$ es einen Zweck oder Grund gibt, wenn jemand ein Besitztum diesem und nicht jenem Menschen überträgt; daß man gewöhnlich erkennen kann, was der Uberträger zu gewinnen glaubte, an welchen Zielen er mitzuwirken glaubte usw. Da in einer kapitalistischen Gesellschaft die Menschen Besitztümer an andere oft im Einklang damit übertragen, wieviel ihnen nach ihrem Empfinden diese anderen nützen, ist die durch die einzelnen Ubertragungen zustandekommende Struktur weitgehend vernünftig und einsichtig." (151)

Es besteht jedoch kein Zweifel, daß die Menschen in der sozialen Realität keines- , wegs stets wohlüberlegt und vernünftig handeln und daß sie die Konsequenzen ihres Handelns nur zu einem kleinen Teil überschauen. Die Menschen können sich irren, sind sich oft gar nicht über ihre Ziele im klaren und jede ihrer Handlungen zieht Nebenfolgen nach sich, die sie nicht beabsichtigten und die sie häufig auch nicht voraussehen konnten. Die Unterstellung, die Menschen handelten bei Übertragungsvorgängen im großen und ganzen rational und sie seien sich aller Folgen ihres Handelns voll bewußt, ist daher in hohem Maße irreal. Da diese Unterstellung jedoch eine notwendige Voraussetzung für Nozicks Schlußfolgerung ist, daß sich aus der moralischen Zulässigkeit von Übertragungsakten zwingend auch die Gerechtigkeit der Ergebnisse derselben ergibt, ist dieser Schluß - wie Cohen völlig zu Recht feststellt - einfach nicht gültig (Cohen 1977,9):

„Nozick sagt, eine Ubertragung sei frei von Ungerechtigkeit, wenn ihr jede betroffene Partei zustimmt. Vielleicht ist dies so. Darüber hinaus nimmt er jedoch an, die so charakterisierte Ubertragungsgerechtigkeit verbürge - unter der Voraussetzung einer anfänglich gerechten Situation - Gerechtigkeit auch der Verhältnisse, die daraus resultieren. ... Und dies ist fraglich. Mit Bezug auf jede Person, die einer Übertragung zustimmt, können wir fragen: würde sie ibr auch zugestimmt haben, wenn sie gewußt bätte, was ibr Ergebnis sein würde? Da die Ant- 
wort negativ sein kann, ist es alles andere als evident, daß die Übertragungsgerechtigkeit, wie beschrieben, die Gerechtigkeit auf ihre Ergebnisse vererbt. Vielleicht aber ist das der Fall, wenn die Antwort positiv ist."

Cohen meint daher, daß wir, um zu einem angemessenen Prinzip der Übertragungsgerechtigkeit zu kommen, welches - anfänglich gerechte Verhältnisse vorausgesetzt - von der Gerechtigkeit der Übertragungsvorgänge auf die Gerechtigkeit der daraus resultierenden Verhältnisse zu schließen erlaubt, Nozicks Leitsatz folgendermaßen verschärfen müssen (Cohen 1977, 9):

„Alles, was aus gerechten Verhältnissen als Ergebnis freiwilliger Transaktionen resultiert, denen alle Beteiligten auch dann zugestimmt hätten, wenn sie gewußt hätten, was die Ergebnisse ihrer Transaktionen sein würden, ist selbst gerecht."

Es versteht sich von selbst, daß - wenn wir diesen Satz als Prinzip der Übertragungsgerechtigkeit annehmen - Nozicks Auffassung, freiwillige Übertragungsvorgänge allein seien ein zureichendes Medium des (abgeleiteten) Erwerbs moralischer Rechte und Pflichten, jede Grundlage entzogen ist.

\section{Anmerkungen}

1 Dt.: Anarchie, Staat, Utopia, München o.J.(1976), aus dem Amerikanischen von Hermann Vetter. Seitenhinweise im Text dieses Aufsatzes beziehen sich, wenn nicht anders angegeben, auf die deutsche Ausgabe von Nozicks Buch.

2 Eine genaue Analyse und Kritik von Nozicks Theorie der Entstehung des Minimalstaates aus dem Naturzustand gibt Kliemt 1980; siehe auch Wolff 1977, Lübbe 1978, Kliemt 1979.

3 Zu den Begriffen der „Anwendbarkeit" ("applicability“) und „Handhabbarkeit" ("practicability") eines Moralkodex siehe Körner 1976, S. 27 ff.

4 Zum Folgenden siehe: Locke 1690, 2. Abhandlung, 5. Kap., \$\$25-50, $215 \mathrm{ff}$.

5 Kants genaue Formulierung in der "Grundlegung zur Metaphysik der Sitten" lautet bekanntlich: „Handle so, daß du die Menschheit, sowohl in deiner Person, als in der Person eines jeden anderen, jederzeit zugleich als Zweck, niemals bloß als Mittel brauchst." (Kant $1785,61$.

6 Kant betont ausdrücklich, sein Prinzip sei nicht nur negativ zu vestehen, in dem Sinne, daß es uns verbietet, den vernünftigen Willen anderer Menschen zu mißachten, sondern es verlange auch positiv, daß wir die Glückseligkeit anderer Menschen als vernünftige Wesen zu befördern trachten sollen. Ein weiteres Mißverständnis von Kants Prinzip bedeutet es, wenn Nozick meint, der Begriff des Zwecks umfasse jede beliebige freie Willensäußerung, also jeden subjektiven $Z$ weck von Menschen. Kant hatte vielmehr so etwas wie „objektive“" Zwecke im Auge, die, ,wir mögen $Z$ wecke haben, welche wir wollen, als Gesetz die oberste einschränkende Bedingung aller subjektiven Zwecke ausmachen" sollen (siehe Kant 1785 , $61 \mathrm{ff}$.; vgl. auch Paton 1947,199ff). Kants Prinzip ist zweifelsohne nicht sehr klar und es wirft gewiß viele Probleme auf, soviel aber läßt sich mit Sicherheit sagen, daß Nozick es nicht verstanden hat. Vgl. hierzu auch Norton 1976/77, $117 \mathrm{ff}$.

\section{Bibliographie}

Arrow, K. J. (1978), Nozick's Entitlement Theory of Justice, Philosophia 7, 265-279

Cohen, G. A. (1977), Robert Nozick and Wilt Chamberlain: How Patterns Preserve Liberty, Erkenntnis 11, 5-23 
Kant, I. (1785), Grundlegung zur Metaphysik der Sitten, zitiert nach: Kant, Werke in zwölf Bänden, hrsg. von W. Weischedel, Bd. VII, Frankfurt/M. 1968.

Kliemt, H. (1979), John Rawls' Theorie der Gerechtigkeit und Robert Nozick's Theorie des Minimalstaates, Okonomische Theorie der Politik, hrsg. von C. Hillinger u. M. J. Holler, München 1979, 196-223

- (1980), Zustimmungstbeorien der Staatsrecbtfertigung, Freiburg-München

Körner, St. (1976), Experience and Conduct, Cambridge

La Follette, H. (1978), Why Libertarianism Is Mistaken, Justice and Economic Distribution, hrsg. von J. Arthur und W. H. Shaw, Englewood Cliffs, N. J. 1978, 194-206

Locke, J. (1690), Two Treatises of Government, dt.: Zwei Abbandlungen über die Regierung, hrsg. von W. Euchner, Frankfurt/M. 1977

Lübbe, G. (1978), Robert Nozicks Naturrech tsidealismus. Zu einer Karikatur des klassischen Liberalismus, Rechtstbeorie 9, 217-228

Nagel, T. (1975), Libertarianism Without Foundations, The Yale Law Journal 85, 136-149

Norton, D. L. (1976/77), Individualism and Productive Justice, Etbics 87, 113-125

Nozick, R. (1974), Anarchy, State, and Utopia, New York; dt.: Anarcbie, Staat, Utopia, München o.J.(1976)

Paton, H.J. (1947), The Categorical Imperative, London; dt.: Der Kategorische Imperativ, Berlin 1962

Rawls, J. (1971), A Theory of Justice, Cambridge, Mass., dt.: Eine Theorie der Gerechtigkeit, Frankfurt/M. 1975

Ryan, C. C. (1976/77), Yours, Mine, and Ours: Property Rights and Individual Liberty, Ethics 87, 126-141

Scanlon, T.M. (1976/77), Nozick on Rights, Liberty, and Property, Pbilosopby \& Public Affairs 6, 3-25

Singer, P. (1978), Rights and the Market, Justice and Economic Distribution, hrsg. von J. Arthur u. W. H. Shaw, Englewood Cliffs, N. J. 1978, 207-221

Thomson, J. J. (1977), Some Ruminations on Rights, Arizona Law Review 19, 45-60

Wolff, R.P. (1977), Robert Nozick's Derivation of the Minimal State, Arizona Law Review 19, 7-30 